\title{
A Systematic Clinical-mimetic Qi Deficiency Syndrome Model in Traditional Chinese Medicine and LDHb/MicroRNA-130a-3p Associated with Syndrome Differentiation
}

\author{
Jincheng Chen ${ }^{1,2}$, Dennis Chang ${ }^{3}$, Junguo Ren², Chengren Lin², Bensoussan Alan ${ }^{3}$, Jianxun Liu² \\ ${ }^{I}$ China Academy of Chinese Medical Sciences, China, ${ }^{2}$ Research \& Development Centre, Xiyuan Hospital of China \\ Academy of Chinese Medical Sciences, China, ${ }^{3}$ National Institute of Complementary Medicine, University of Western \\ Sydney, Penrith, New South Wales, Australia
}

Background: As the key concept, Syndrome defined by a comprehensive analysis of the four-diagnostic methods, which based on the clinical characters and logical reasoning. In addition, integrate syndrome differentiation with molecular level methods would be imperative. Currently, there is no adequate animal Qi deficiency (QD) syndrome model and systematic evaluation, and no biological features has been identified. In the present study, a typical QD syndrome model developed in rodents and a systematic evaluation established with the clinical-approach four-diagnosis. Based on the genomic direction from clinical samples, the circulating extracellular microRANs and the objective proteins as the "fifth diagnosis" to be examined.

Methods and Results: To evaluate the equivalence between the character of syndrome model and the major clinical features. We developed a QD syndrome model with the treatment of chronic paradoxical sleep deprivation to mirror facts of QD in rodents. The whole behaviour, body weight variation, locomotive activity, Echocardiography, exhaustive time, tongue and pulse diagnosis were measured, which could induce the characters and signs of QD syndrome with physically and mentally fatigued, palpitation, short of breath, pale tongue and weak pulse, and so forth. Finally, the circulating microRNA changes of QD in normal control and syndrome model were compared on the differential microRNAs profiles in health controls and two syndromes of coronary heart disease (CHD) patients. miR-130a-3p was significantly increased and the objective protein LDHb expression upregulated in QD syndrome compared with normal controls, implicating that energy metabolism disorder were involved in biological basis of syndrome, and molecular level as the "the fifth diagnosis" to identify the syndrome model correctly.

Conclusions: These studies established a QD syndrome rodent model, and a systematic evaluation of "five-diagnosis" composed of clinical-mimetic four-diagnostic methods and LDHb/microRNA-130a-3p molecular level micro-diagnostic. This research provides a key and foundation method to recognize syndrome, to perform drugs and disease research in TCM. 